\title{
The Efficacy and Safety of 12 Weeks of Sofosbuvir and Ledipasvir versus Sofosbuvir, Ledipasvir, and Ribavirin in Patients with Chronic Hepatitis C, Genotype 1, Who Have Cirrhosis and Have Failed Prior Therapy: A Systematic Review and Meta-Analysis
}

\author{
William Stokes, ${ }^{1}$ Carol Fenton, ${ }^{2}$ Fiona Clement, ${ }^{2}$ Matthew James, ${ }^{1,2}$ \\ Paul Ronksley, ${ }^{2}$ and Karen L. Tang ${ }^{1,2}$ \\ ${ }^{1}$ Department of Medicine, University of Calgary, Foothills Medical Centre, 1403 29th St. NW, Calgary, AB, Canada T2N 2 T9 \\ ${ }^{2}$ Department of Community Health Sciences, University of Calgary, Teaching, Research and Wellness Building, 3280 Hospital Drive \\ NW, Calgary, AB, Canada T2N 4N1
}

Correspondence should be addressed to William Stokes; wstokes@mun.ca

Received 7 September 2016; Revised 13 January 2017; Accepted 26 January 2017; Published 6 March 2017

Academic Editor: Kevork M. Peltekian

Copyright (C) 2017 William Stokes et al. This is an open access article distributed under the Creative Commons Attribution License, which permits unrestricted use, distribution, and reproduction in any medium, provided the original work is properly cited.

Background. The recommended therapy for patients with chronic hepatitis $\mathrm{C}$ (CHC), genotype 1, who have cirrhosis and have failed prior therapy is 12 weeks of sofosbuvir (SOF), ledipasvir (LDV), and ribavirin (RBV). This recommendation is based on expert opinion, and the efficacy of 12 weeks of SOF/LDV compared to SOF/LDV/RBV in this patient population has not yet been established. Methods. We conducted a systematic review and meta-analysis. Two investigators independently searched electronic databases and relevant conference proceedings for randomized controlled trials comparing rates of sustained virologic response 12 weeks after therapy (SVR12) when using 12 weeks of SOF/LDV versus 12 weeks of SOF/LDV/RBV in patients with CHC, genotype 1, who have cirrhosis and failed previous therapy. Results. Our search strategy yielded 596 studies of which four met criteria for inclusion. The pooled RR of not achieving SVR12 with SOF/LDV versus SOF/LDV/RBV was 1.21 (95\% CI: 0.42-3.48). Adverse events were lower in the SOF/LDV compared to the SOF/LDV/RBV arms (pooled RR: 0.11, 95\% CI: 0.04-0.29). Conclusions. Our findings suggest that 12 weeks of SOF/LDV cannot be considered noninferior to 12 weeks of SOF/LDV/RBV to achieve SVR12 in patients with $\mathrm{CHC}$ who have cirrhosis and failed prior therapy.

\section{Introduction}

Over 210,000 Canadians are estimated to be currently living with chronic hepatitis $\mathrm{C}(\mathrm{CHC})$ infections [1]. To date, liver failure due to $\mathrm{CHC}$ is still the number one indication for liver transplant in Canada [2]. The number of Canadians with advanced $\mathrm{CHC}$ that develop cirrhosis is increasing annually and is expected to peak between 2031 and 2035 at approximately 40,000 per year [3]. Hepatitis C-related healthcare costs are forecasted to increase by $60 \%$ between 2013 and 2032, mostly due to cirrhosis and associated complications [3].
In Canada, 12 weeks of sofosbuvir and ledipasvir is the first-line therapy for patients without comorbidities with $\mathrm{CHC}$, genotype 1 , who are treatment naïve. However, certain characteristics put patients at higher risk of treatment failures. This includes patients who have previously failed therapy, or "null responders," and those who have cirrhosis. For both patients with cirrhosis and those who have failed previous therapy, current guidelines recommend the use of either 12 weeks of SOF/LDV/RBV or 24 weeks of SOF/LDV alone [4]. These guidelines are based only on expert opinion, as there was limited evidence available on the effectiveness of the new medications in these populations. 
In the ION2 study, 12 weeks of SOF/LDV, 12 weeks of $\mathrm{SOF} / \mathrm{LDV} / \mathrm{RBV}$, and 24 weeks of SOF/LDV were compared to each other for patients with hepatitis C, genotype 1, who have cirrhosis and failed prior therapy [5]. In this subgroup of patients, SVR12 was found to be $99 \%$ with 24 weeks of LDV/SOF, $86 \%$ with 12 weeks of LDV/SOF, and $82 \%$ with 12 weeks of $\mathrm{LDV} / \mathrm{SOF} / \mathrm{RBV}$. The study therefore suggested that 24 weeks of SOF/LDV is better than 12 weeks of SOF/LDV or 12 weeks of SOF/LDV/RBV in patients with $\mathrm{CHC}$, genotype 1, who have cirrhosis and failed prior therapy. However, the subsequent SIRIUS study refuted ION2's results by demonstrating similarly high rates of SVR12 between 24 weeks of SOF/LDV (97\%) and 12 weeks of SOF/LDV/RBV (96\%) [6]. As a result, current guidelines recommend either 24 weeks of SOF/LDV or 12 weeks of SOF/LDV/RBV for patients with $\mathrm{CHC}$, genotype 1, who have cirrhosis and failed prior therapy [7]. Unfortunately, the SIRIUS trial did not include 12 weeks of SOF/LDV. Therefore, it is unclear whether 12 weeks of SOF/LDV/RBV is superior to 12 weeks of SOF/LDV. Other studies comparing 12 weeks of SOF/LDV versus $\mathrm{SOF} / \mathrm{LDV} / \mathrm{RBV}$ in patients with hepatitis $\mathrm{C}$, genotype 1 , who have cirrhosis and failed prior therapy show results that suggest that 12 weeks of SOF/LDV versus SOF/LDV/RBV in this population may have similar results, but no pooled systematic review and meta-analysis has been done to determine whether any differences among the two treatments exist $[5,8-$ $10]$.

We undertook a systematic review and meta-analysis of randomized controlled trials to determine the efficacy of 12week therapy with SOF/LDV compared to SOF/LDV/RBV in achieving SVR12 in cirrhotic patients with chronic hepatitis C, genotype 1, who have previously failed therapy. Our secondary objective was to determine the risk of adverse events for both treatment regimens.

\section{Methods}

2.1. Search Strategy. We performed a systematic review and meta-analysis using a predetermined protocol and in accordance with PRISMA standardized reporting guidelines [11]. Two investigators (WS and CF) independently searched electronic databases from inception to November 8, 2015, including PubMed, the Central Registry of Controlled Trials of the Cochrane Library, MEDLINE, EMBASE, clinicaltrials.org, and Science Citation Index Expanded. No language restrictions were applied. Our search strategy combined the names and alternate names of sofosbuvir and ledipasvir using the Boolean operator AND to map (search by keyword) and explode (search by subject heading where appropriate). Alternative names of sofosbuvir and ledipasvir, or their active metabolites, were combined using the Boolean operator OR. Harvoni ${ }^{\circledR}$, the combination brand name for ledipasvir/sofosbuvir, was also searched and combined with the above terms using the Boolean operator OR (Supplemental Appendix A, available online at https://doi.org/10.1155/2017/6468309). Our search was broad in order to capture all relevant publications given the relative novelty of sofosbuvir and ledipasvir combination therapy.
Conference proceedings from the American College of Gastroenterology Annual Scientific Meetings, Annual Meetings of the American Association for the Study of Liver Diseases, and Annual Meetings of the Infectious Diseases Society of America were also searched for relevant abstracts. Conference proceedings were searched from 2010 onwards as ledipasvir was not chemically identified until 2013 [12]. Finally, experts in the field and Gilead Sciences were contacted for other published work and reference lists of all relevant publications were manually searched.

2.2. Identification of Articles for Systematic Review and MetaAnalysis. All identified abstracts were screened for fulltext review by two investigators (WS and CF). Interrater agreement was measured using the kappa statistic. Disagreements were resolved by consensus. Articles were included if they were randomized clinical trials presenting original data comparing the combinations of 12 weeks of SOF/LDV and SOF/LDV/RBV in individuals with chronic hepatitis $\mathrm{C}$, genotype 1 . We analyzed genotypes $1 \mathrm{a}$ and $1 \mathrm{~b}$ together since the two could not be independently assessed for patients with cirrhosis who failed previous therapy without access to individual patient data. Trials that did not have study arms of cirrhotic patients who failed previous therapy were excluded. Trials that did not measure sustained virologic response at 12 weeks after treatment were also excluded.

2.3. Data Extraction and Quality Assessment. Data extraction was performed independently by the same two investigators (WS and CF). Data extracted included study characteristics (sites involved, year, location, and inclusion/exclusion criteria), patient characteristics (genotype 1 subtype, age, sex, percentage of patients who failed previous therapy, percentage of patients with cirrhosis, and percentage of patients with nonCC interleukin 28B gene locus (IL28B) genotypes), treatment characteristics (doses and duration), and outcome characteristics (number of SVR12, dropouts, viral breakthrough, viral relapse, and adverse events). Study quality was also independently assessed using the Cochrane Collaboration tool for assessing risk of bias [13]. The Cochrane Collaboration elements for assessing risk of bias include random sequence generation, allocation concealment, blinding, attrition bias, reporting bias, and other biases (such as conflicts of interest through industry funding).

2.4. Data Synthesis and Analysis. Failure to achieve SVR12 among cirrhotic patients who failed previous CHC therapy with IFN based or protease inhibitor based regimens was assessed using relative risk (RR), with the reference group being patients receiving 12 weeks of LDV/SOF/RBV. Study subjects lost to follow-up were included as virologic failures. Pooled estimates were derived using DerSimonian and Laird random effects models given the observed differences in patient characteristics between studies. Pooled RRs were visually demonstrated using forest plots and heterogeneity among studies was assessed using the $I^{2}$ and Cochran $Q$ statistics. The relative risks of adverse effects of 12 weeks of SOF/LDV versus SOF/LDV/RBV were also pooled using random effects models, with patients receiving 12 weeks 


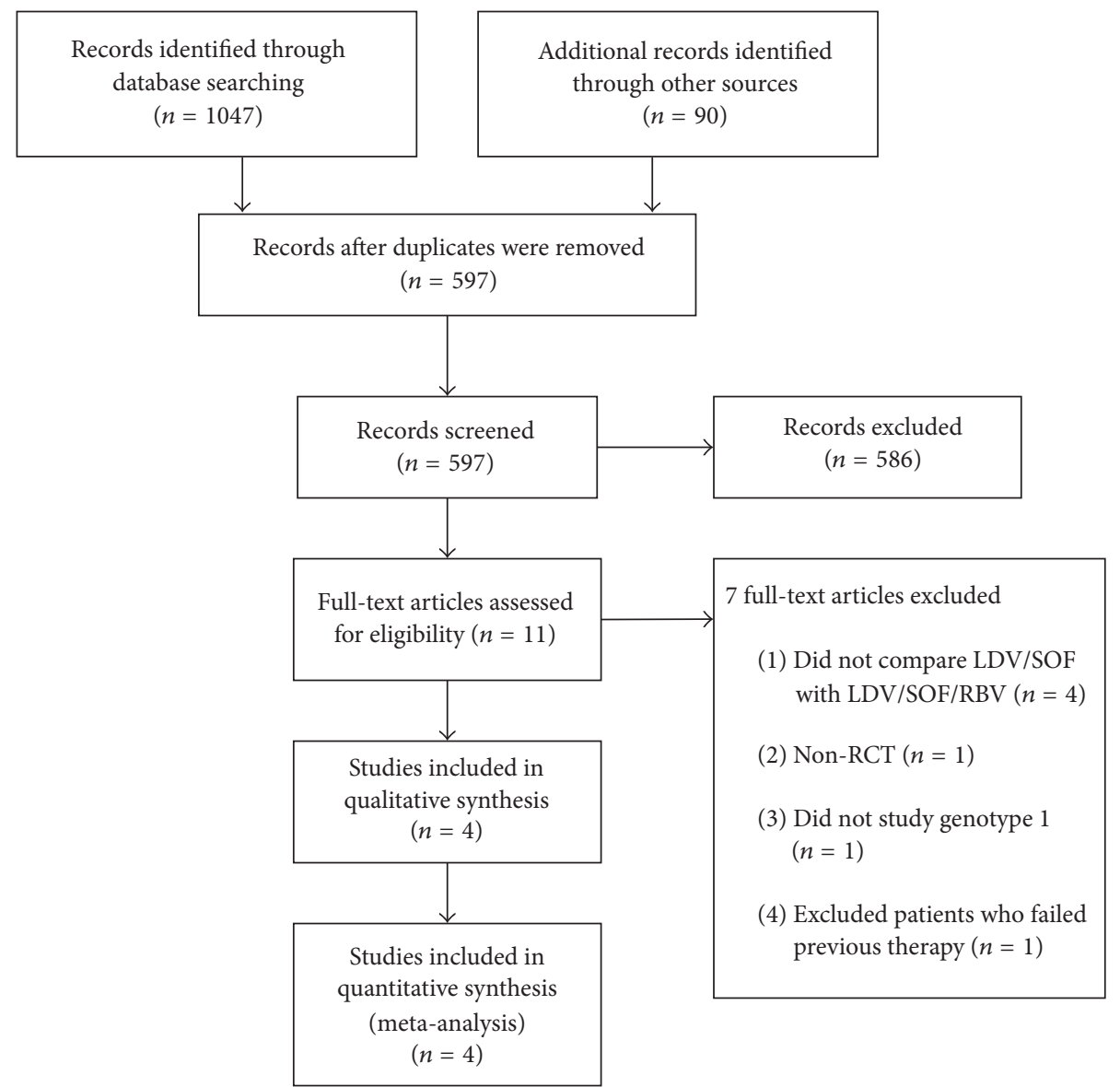

FIgURE 1: Search strategy results using PRISMA flow diagram [26].

of LDV/SOF/RBV as the reference group. As a sensitivity analysis for all pooled estimates, fixed effect models were generated to assess the robustness of the findings. Finally, small study effects, potentially indicative of publication bias, were assessed visually through a funnel plot and tested for using Begg and Mazumdar's rank correlation test for asymmetry [14]. A significant statistical test $(p<0.05)$ or observed funnel plot asymmetry suggests small study effects that may potentially be caused by publication bias. All analyses were conducted using STATA 14.0 (StataCorp., College Station, TX, USA).

\section{Results}

3.1. Search Results. Our search strategy yielded 597 abstracts, of which 586 were excluded for not meeting inclusion/ exclusion criteria (Figure 1). Subsequently, 11 papers were included for full-text review, of which 4 papers met inclusion criteria for inclusion in the systematic review [5, 810]. Agreement was excellent between the two investigators (kappa statistic $=1.0$ for the 4 included papers).

3.2. Study Characteristics. Characteristics of previously treated subjects from the 4 included studies are provided in Table 1. Two studies were phase II trials and two were phase
III. Studies varied widely in terms of geographic location, hepatitis $C$ genotype 1 subtypes, and patient characteristics. Studies were carried out in USA, New Zealand, or Japan. Study participants were, on average, middle aged. Men represented the majority of participants in all studies except for one [10]. All studies included participants with cirrhosis, but in frequencies among the previously treated subgroups ranging from $20 \%$ to $100 \%$. Furthermore, all studies had different proportions of subgenotype la or $1 \mathrm{~b}$ included, ranging from $13 \%$ to over $96 \%$ genotype 1a. Among all studies, patients with significant comorbidities including human immunodeficiency virus, hepatitis $\mathrm{B}$, and decompensated cirrhosis were excluded.

All trials used the same dose of SOF/LDV/RBV except for Mizokami et al., who used a lower RBV dose as recommended by the manufacturers for Japanese patients. Outcome measurements were consistent across studies and were defined as undetectable HCV after 12 weeks of therapy using COBAS TaqMan HCV test for HCV RNA detection. Undetectable $\mathrm{HCV}$ was defined as $<25 \mathrm{IU} / \mathrm{mL}$ for all studies except for the ELECTRON trial in which undetectability was defined as $<43 \mathrm{IU} / \mathrm{mL}$. All studies had fewer than $2 \%$ of subjects lost to follow-up [9].

Cochrane Risk of Bias Assessment yielded similar risks for all included studies (Table 2). Studies were generally high 
TABLE 1: Study characteristics of the previously treated subgroups within included studies.

\begin{tabular}{|c|c|c|c|c|}
\hline Study & LONESTAR [8] & ELECTRON [9] & ION2 [5] & Mizokami et al. [10] \\
\hline Publication year & 2014 & 2014 & 2014 & 2015 \\
\hline Sample size of previously treated subjects given 12 weeks of therapy & 40 & 19 & 220 & 175 \\
\hline Cirrhosis & $55 \%$ & $100 \%$ & $20 \%$ & $29 \%$ \\
\hline Geographical location & USA & New Zealand & USA & Japan \\
\hline Funding source & Industry & Industry & Industry & Industry \\
\hline Number lost to follow-up & 0 & 0 & 0 & 0 \\
\hline Average age (years) & 53 & 59 & 57 & $35 \%>$ age 65 \\
\hline Male gender & $73 \%$ & $95 \%$ & $66 \%$ & $43 \%$ \\
\hline Genotype la & $86 \%$ & $81 \%$ & $79 \%$ & $4 \%$ \\
\hline Genotype $1 b$ & $14 \%$ & $19 \%$ & $21 \%$ & $96 \%$ \\
\hline Black race & $10 \%$ & $0 \%$ & $18 \%$ & $0 \%$ \\
\hline Mean BMI $\left(\mathrm{Kg} / \mathrm{m}^{2}\right)$ & 31.5 & 29.2 & 29 & $62 \%<25 \mathrm{Kg} / \mathrm{m}^{2}$ \\
\hline Mean HCV RNA $\left(\log _{10} \mathrm{IU} / \mathrm{mL}\right)$ & 6.3 & 6.4 & 6.5 & $87 \%>800,000 \mathrm{IU} / \mathrm{mL}$ \\
\hline
\end{tabular}

TABLE 2: Cochrane Risk of Bias Assessment for included studies.

\begin{tabular}{|c|c|c|c|c|}
\hline & LONESTAR & ELECTRON & ION2 & Mizokami \\
\hline \multicolumn{5}{|c|}{ Random sequence generation } \\
\hline \multicolumn{5}{|l|}{ Allocation concealment } \\
\hline \multicolumn{5}{|l|}{ Blinding } \\
\hline \multicolumn{5}{|c|}{$\begin{array}{l}\text { Attrition bias/incomplete outcome } \\
\text { data }\end{array}$} \\
\hline \multicolumn{5}{|c|}{ Reporting bias/selective reporting } \\
\hline Other biases (funding) & & & & \\
\hline
\end{tabular}

+ Low risk of bias

- High risk of bias

in quality, except for the presence of a significant risk of bias associated with nonblinding and for being funded and conducted by the makers of sofosbuvir and ledipasvir (Gilead Sciences).

3.3. Sustained Virologic Response. Overall, failure to achieve SVR12 occurred in $0-30 \%$ and $0-19 \%$ of cirrhotic patients who failed previous therapy who underwent 12 weeks of therapy with SOF/LDV and SOF/LDV/RBV, respectively. Of note, the ELECTRON trial was an outlier among study results, having 30\% SVR12 failure rates in the LDV/SOF group compared to the $0 \%$ to $14 \%$ among the other studies [9].

Among cirrhotic patients who failed previous therapy, the pooled RR of not achieving SVR12 after completing 12 weeks of SOF/LDV therapy compared to 12 weeks of SOF/LDV/RBV was 1.21 (95\% CI: 0.42-3.48) (Figure 2). Unfortunately, SVR for genotype subtype la could not be adequately assessed among studies without access to individual patient data. To determine whether genotype subtype had a significant impact among cirrhotic patients who failed previous therapy, we performed sensitivity analysis by excluding Mizokami et al.s study, as $96 \%$ of its study participants were genotype $1 \mathrm{~b}$ compared to $13-21 \%$ among the other studies [10]. Excluding Mizokami et al's study yielded minimal changes, with the pooled RR of not achieving SVR12 increasing slightly to 1.39 (95\% CI: 0.39-4.97). Though with limited capacity of detecting heterogeneity with low number of studies, in the overall results, the Cochran $Q$ test statistic was 0.516 indicating homogeneity among studies. $I^{2}$, despite being also limited by small sample size, was $0 \%$. Fixed effect modeling did not change the statistical significance of any result.

3.4. Adverse Events. We were unable to assess adverse events in cirrhotic patients only, so we have presented the pooled overall results for these outcomes. Adverse events were significantly more common in patients who received SOF/LDV/RBV compared to those who received SOF/LDV. The pooled relative risk for having any adverse event in LDV/SOF compared to LDV/SOF/RBV was 0.11 (95\% CI: 0.04-0.29) (Table 3). Differences in adverse events were observed for those known to be associated with ribavirin, including fatigue/asthenia, rash, irritability, cough/ bronchitis, and anemia. Three patients in the SOF/LDV/RBV arms discontinued study drugs due to adverse events. These 
TABLE 3: Combined adverse events for SOF/LDV and SOF/LDV/RBV and their associated pooled relative risks, with SOF/LDV/RBV as the reference group.

\begin{tabular}{|c|c|c|c|}
\hline Adverse effect & SOF/LDV $(N=328)$ & SOF/LDV/RBV $(N=344)$ & Pooled RR $(95 \%$ CI $)$ \\
\hline Any side effect & 223 & 523 & $0.11(0.04-0.29)$ \\
\hline Discontinuation because of adverse effect & 0 & 3 & $0.66(0.11-3.84)$ \\
\hline Serious & 5 & 7 & $1.09(0.64-1.85)$ \\
\hline Fatigue/asthenia & 33 & 68 & $0.57(0.39-0.83)$ \\
\hline Rash & 2 & 18 & $0.20(0.06-0.66)$ \\
\hline Irritability & 2 & 18 & $0.19(0.05-0.70)$ \\
\hline Cough/bronchitis & 7 & 23 & $0.39(0.17-0.88)$ \\
\hline Anemia & 3 & 45 & $0.11(0.04-0.24)$ \\
\hline HA & 44 & 61 & $0.97(0.77-1.24)$ \\
\hline Insomnia & 10 & 27 & $0.67(0.40-1.10)$ \\
\hline Nausea & 19 & 43 & $0.73(0.51-1.05)$ \\
\hline Diarrhea & 7 & 8 & $1.16(0.71-1.90)$ \\
\hline URTI & 7 & 23 & $0.50(0.21-1.19)$ \\
\hline Muscle spasm & 1 & 10 & $0.29(0.08-1.06)$ \\
\hline Arthralgia & 7 & 20 & $0.66(0.36-1.20)$ \\
\hline Dry skin & 0 & 10 & $0.26(0.04-1.73)$ \\
\hline Dizziness & 3 & 13 & $0.52(0.21-1.30)$ \\
\hline Dyspnea & 0 & 21 & $0.11(0.01-1.59)$ \\
\hline $\mathrm{Hgb}<8.5 \mathrm{~g} / \mathrm{dL}$ & 1 & 9 & $0.58(0.07-4.750)$ \\
\hline Lymphocytes $<350$ per $\mathrm{mm}^{3}$ & 0 & 1 & $\mathrm{~N} / \mathrm{A}^{*}$ \\
\hline Neutrophils $500-750$ per $\mathrm{mm}^{3}$ & 2 & 0 & $\mathrm{~N} / \mathrm{A}^{*}$ \\
\hline Platelets $25-50$ per $\mathrm{mm}^{3}$ & 2 & 0 & $1.51(0.85-2.68)$ \\
\hline
\end{tabular}

${ }^{*}$ Pooled result not possible as adverse event recorded in one study only.

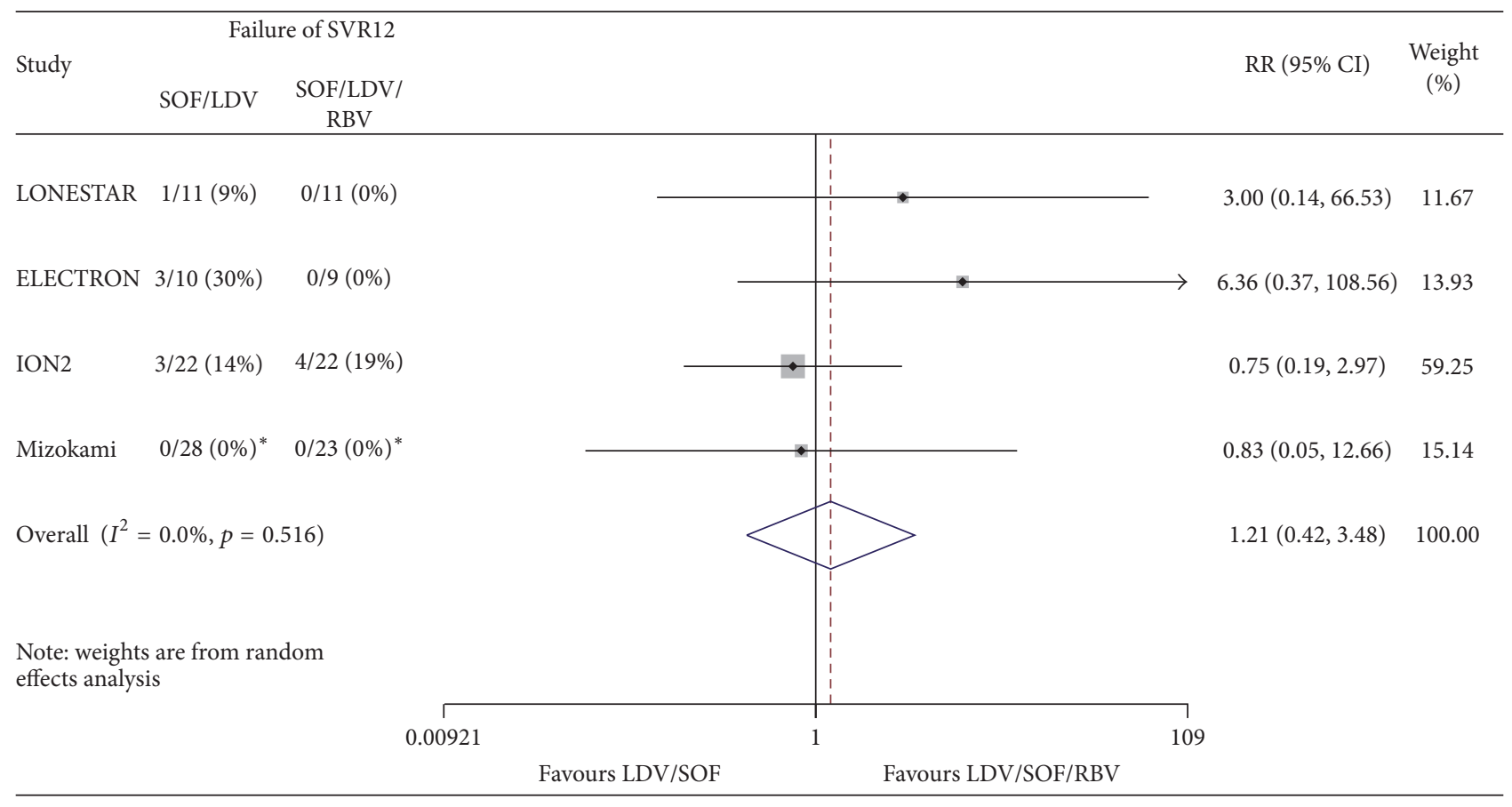

${ }^{*}$ Continuity correction of 1 applied for meta-analysis.

FIGURE 2: Forest plot of relative risks of not achieving SVR12 with 12 weeks of SOF/LDV versus SOF/LDV/RBV in chronic hepatitis C, genotype 1 , of cirrhotic patients who have failed previous therapy. 


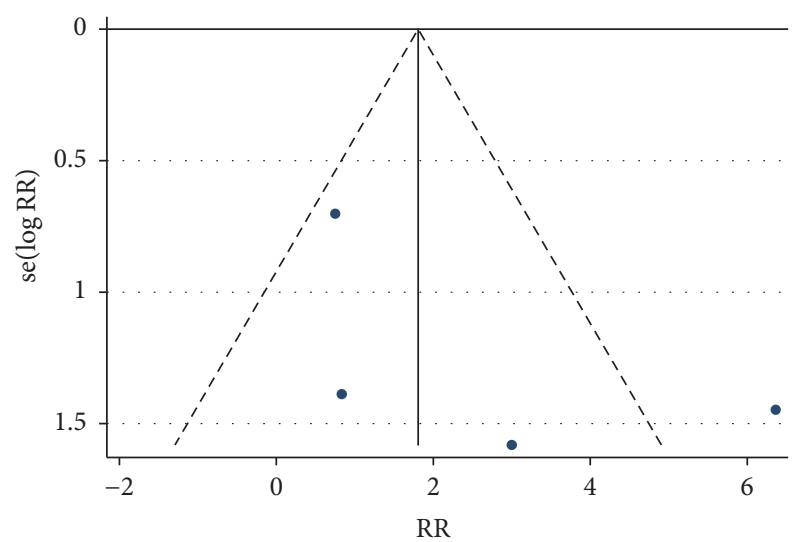

FIgURE 3: Assessment of publication bias using funnel plot with pseudo $95 \%$ confidence limits.

adverse events included cardiac arrest, ribavirin-associated morbilliform drug eruption, and peritonitis secondary to perforated diverticulum, of which the two former events were felt to be related to study treatment. Serious adverse events were higher in SOF/LDV/RBV versus the SOF/LDV arms but differences were not statistically significant. A total of four serious adverse events were thought to be related to study therapy and all occurred in the SOF/LDV/RBV arms. These were hemolytic anemia, nonfatal myocardial infarction, and the aforementioned morbilliform rash and cardiac arrest.

3.5. Publication Bias. Although assessment for publication bias was conducted, interpretation of results was significantly limited by the small number of studies involved. The associated funnel plot shows visual asymmetry, suggesting that publication bias may be present (Figure 3). The funnel plot also demonstrates an outlier, which corresponds to the ELECTRON trial [9].

\section{Discussion}

The results of our systematic review and meta-analysis suggest that 12 weeks of SOF/LDV cannot be considered noninferior to 12 weeks of SOF/LDV/RBV in achieving SVR12 in patients with hepatitis C, genotype 1, who have cirrhosis and have previously failed therapy. When examining the pooled point estimate, the pooled effect size is in keeping with over a $20 \%$ increase in risk of failure to achieve remission. Also, the range of plausible effect illustrates the imprecision of the pooled estimate, with as much as a 3 -fold potential increase in risk based on the pooled effect seen from the existing studies, beyond an acceptable threshold for SOF/LDV to be considered noninferior. Our results therefore support continued adherence to recent guidelines, which recommend the preferential use of 12-week SOF/LDV/RBV over 12-week SOF/LDV alone in this subgroup until more research has been conducted [4].

Results from our systematic review indicate that RCTs examining 12 weeks of LDV/SOF versus LDV/SOF/RBV in patients with hepatitis $\mathrm{C}$, genotype 1 , who have cirrhosis and previously failed therapy are of moderate quality. All studies were nonblinded, which may be due to requirements to monitor adverse effect profiles, including anemia. However, there is concern regarding pharmaceutical company Gilead Sciences' substantial role in funding and coordinating the studies. While steps are often taken to minimize bias in company sponsored trials, it is known that these trials tend to favour the company's product compared to independently conducted trials regardless of trial methodology [15-17].

As expected, adverse events were higher among SOF/LDV/RBV compared to SOF/LDV and were due to adverse events that are typically associated with ribavirin treatment such as anemia, fatigue, rash, and cough. Serious events, including those associated with discontinuation of study drug, were more likely to occur with SOF/LDV/RBV treatment but the difference was not statistically significant between groups. Use of SOF/LDV/RBV over SOF/LDV may further increase risk of nonadherence by increasing pill burden and pill frequency [18]. Determining noninferiority between SOF/LDV/RBV and SOF/LDV is therefore an important avenue to continue researching since eliminating RBV from treatment regimens has potential to improve adherence rates in real world settings.

Our study is the first systematic review and meta-analysis of randomized controlled trials comparing 12 weeks of $\mathrm{SOF} / \mathrm{LDV}$ versus 12 weeks of SOF/LDV/RBV in patients with hepatitis $\mathrm{C}$, genotype 1 , who have cirrhosis and have failed previous therapy. Our study does have several limitations, however. We were unable to prove noninferiority given our very wide confidence intervals in the pooled effect estimates. Estimating 95\% success rates for LDV/SOF and $\mathrm{LDV} / \mathrm{SOF} / \mathrm{RBV}$ and using a noninferiority limit of $5 \%$, a clinical trial with sample size of 652 patients (326 per arm) would be needed to prove noninferiority with $90 \%$ power and significance level of 5\% [19]. Moreover, trials included in our study differed widely. Although we were able to extract data for cirrhotic patients who failed previous therapy, subgroup analysis (such as for the subgroup with HCV genotype subtype 1a) was limited without having individual patient data. Individual patient data is important in the context of $\mathrm{HCV}$, where clinical heterogeneity that could influence the outcome of SVR may not be adequately documented at the aggregate level. Therefore, more studies, especially those independent of pharmaceutical involvement, are needed to more accurately compare the degree of efficacy between $\mathrm{LDV} / \mathrm{SOF}$ and LDV/SOF/RBV.

Although newer HCV treatment regimens have emerged, SOF/LDV continues to be relevant in the treatment for $\mathrm{HCV}$, genotype 1 , in patients with cirrhosis who previously failed therapy. Elbasvir/grazoprevir/ribavirin is effective for patients with HCV, genotype 1, who previously failed treatment but requires frequent monitoring in patients with cirrhosis [20]. Sofosbuvir/daclatasvir/ribavirin can be used in cirrhotic patients who previously failed therapy but require longer treatment durations (24 weeks) [21]. The most promising newest $\mathrm{HCV}$ treatment regimen is sofosbuvir/velpatasvir (SOF/VEL), which was approved by Health Canada in July 2016 for the treatment of all genotypes of hepatitis C, including patients with cirrhosis who failed previous therapy [22]. 
Similar to SOF/LDV, SOF/VEL offers once daily dosing for a total of 12 weeks and has a largely benign side effect profile. SOF/VEL has the added benefit of not requiring ribavirin for treatment of genotype 1 cirrhotic patients who failed previous therapy and in fact may be cheaper, though the direct patient cost is not yet fully known [23]. However, SOF/LDV still has several advantages of SOF/VEL in Canada. Currently, SOF/VEL is not yet widely prescribed in Canada since $\mathrm{SOF} / \mathrm{VEL}$ is not covered by most provincial health systems while SOF/LDV is. In addition, cheaper generic versions of SOF/LDV will become available before SOF/VEL since SOF/LDV's patent was approved in 2014 [24], compared to 2016 [25] for SOF/VEL. Therefore, even in the context of new and emerging HCV therapies, SOF/LDV and SOF/LDV/RBC remain important for cirrhotic patients who failed prior therapy. An understanding of the optimal treatment duration and regimen for these two treatment options continues to be crucial.

In conclusion, our study could not determine whether 12 weeks of SOF/LDV is noninferior to 12 weeks of SOF/LDV/RBV for achieving SVR12 in cirrhotic patients who failed previous therapy with chronic hepatitis C, genotype 1. Determining whether 12 weeks of LDV/SOF for cirrhotic patients who have failed previous therapy is as effective as 12 weeks of SOF/LDV/RBV, or the alternatively approved regimen of 24 weeks of SOF/LDV, is an important avenue to continue exploring. Using 12 weeks of SOF/LDV over 24 weeks of LDV/SOF has numerous benefits including substantial cost saving; the shorter duration of therapy may also be critical in patients who are intolerant or ineligible for ribavirin treatment or in whom ribavirin may not be favoured, such as patients at high risk for nonadherence. Further high-quality randomized controlled trials are required to assess the optimal drug regimen and duration in cirrhotic patients with chronic hepatitis $\mathrm{C}$, genotype 1 , who have previously failed therapy.

\section{Disclosure}

An earlier version of this work was presented as a poster at AMMI Canada, CACMID Annual Conference, March 30April 2, 2016.

\section{Competing Interests}

The authors declare that there are no competing interests regarding the publication of this paper.

\section{References}

[1] M. Trubnikov, P. Yan, and C. Archibald, "Estimated prevalence of Hepatitis C virus infection in Canada," Canada Communicable Disease Report CCDR, vol. 40, no. 19, 2011.

[2] Liver Disease in Canada: A Crisis in the Making, http://www .liver.ca/files/PDF/Liver_Disease_Report_2013/Liver_Disease_ in_Canada_-_E.pdf.

[3] R. Myers, M. Krajden, M. Bilodeau, K. Kaita, P. Marotta, and K. Peltekian, "Burden of disease and cost of chronic hepatitis C infection in Canada," Canadian Journal of Gastroenterology and Hepatology, vol. 28, no. 5, pp. 243-250, 2014.
[4] R. P. Myers, A. Ramji, M. Bilodeau, S. Wong, and J. J. Feld, "An update on the management of chronic hepatitis C: consensus guidelines from the Canadian Association for the study of the liver," Canadian Journal of Gastroenterology, vol. 26, no. 6, pp. 359-375, 2012.

[5] N. Afdhal, K. R. Reddy, D. R. Nelson et al., "Ledipasvir and sofosbuvir for previously treated HCV genotype 1 infection," The New England Journal of Medicine, vol. 370, no. 16, pp. 14831493, 2014.

[6] M. Bourlière, J.-P. Bronowicki, V. de Ledinghen et al., "Ledipasvir-sofosbuvir with or without ribavirin to treat patients with HCV genotype 1 infection and cirrhosis non-responsive to previous protease-inhibitor therapy: a randomised, doubleblind, phase 2 trial (SIRIUS)," The Lancet Infectious Diseases, vol. 15, no. 4, pp. 397-404, 2015.

[7] S. Chopra, A. Muir, A. Bisceglie, and A. Bloom, "Treatment regimens for chronic hepatitis $\mathrm{C}$ virus genotype 1," UpToDate, 2015, http://www.uptodate.com/contents/treatment-regimensfor-chronic-hepatitis-c-virus-genotype-1-infection-in-adults.

[8] E. Lawitz, F. F. Poordad, P. S. Pang et al., "Sofosbuvir and ledipasvir fixed-dose combination with and without ribavirin in treatment-naive and previously treated patients with genotype 1 hepatitis $\mathrm{C}$ virus infection (LONESTAR): an open-label, randomised, phase 2 trial," The Lancet, vol. 383, no. 9916, pp. 515-523, 2014.

[9] E. J. Gane, C. A. Stedman, R. H. Hyland et al., "Efficacy of nucleotide polymerase inhibitor sofosbuvir plus the NS5A inhibitor ledipasvir or the NS5B non-nucleoside inhibitor GS9669 against HCV genotype 1 infection," Gastroenterology, vol. 146, no. 3, pp. 736.el-743.el, 2014.

[10] M. Mizokami, O. Yokosuka, T. Takehara et al., "Ledipasvir and sofosbuvir fixed-dose combination with and without ribavirin for 12 weeks in treatment-naive and previously treated Japanese patients with genotype 1 hepatitis C: an open-label, randomised, phase 3 trial," The Lancet Infectious Diseases, vol. 15, no. 6, pp. 645-653, 2015

[11] L. Shamseer, D. Moher, M. Clarke et al., "Preferred reporting items for systematic review and meta-analysis protocols (PRISMA-P) 2015: elaboration and explanation," British Medical Journal, vol. 349, Article ID g7647, 2015.

[12] J. O. Link, J. G. Taylor, L. Xu et al., "Discovery of ledipasvir (GS-5885): a potent, once-daily oral NS5A inhibitor for the treatment of hepatitis C virus infection," Journal of Medicinal Chemistry, vol. 57, no. 5, pp. 2033-2046, 2014.

[13] J. P. Higgins, D. G. Altman, P. C. Gotzsche et al., “The Cochrane Collaboration's tool for assessing risk of bias in randomised trials," BMJ, vol. 343, Article ID d5928, 2011.

[14] C. B. Begg and M. Mazumdar, "Operating characteristics of a rank correlation test for publication bias," Biometrics, vol. 50, no. 4, pp. 1088-1101, 1994.

[15] J. E. Bekelman, Y. Li, and C. P. Gross, "Scope and impact of financial conflicts of interest in biomedical research. A systematic review," JAMA, vol. 289, no. 4, pp. 454-465, 2003.

[16] J. Lexchin, L. A. Bero, B. Djulbegovic, and O. Clark, "Pharmaceutical industry sponsorship and research outcome and quality: systematic review," British Medical Journal, vol. 326, no. 7400, pp. 1167-1170, 2003.

[17] A. Lundh, S. Sismondo, J. Lexchin, O. A. Busuioc, and L. Bero, "Industry sponsorship and research outcome," Cochrane Database of Systematic Reviews, vol. 12, 2012. 
[18] WHO, Adherence to Long-Term Therapies: Evidence for Action, WHO, Geneva, Switzerland, 2003, http://apps.who.int/medicinedocs/pdf/s4883e/s4883e.pdf.

[19] W. C. Blackwelder, "Proving the null hypothesis' in clinical trials," Controlled Clinical Trials, vol. 3, no. 4, pp. 345-353, 1982.

[20] M. Buti, S. C. Gordon, E. Zuckerman et al., "Grazoprevir, elbasvir, and ribavirin for chronic hepatitis $\mathrm{C}$ virus genotype 1 infection after failure of pegylated interferon and ribavirin with an earlier-generation protease inhibitor: final 24-week results from C-SALVAGE," Clinical Infectious Diseases, vol. 62, no. 1, pp. 32-36, 2016.

[21] L. Boglione, S. M. Pinna, C. S. Cardellino et al., "Treatment with daclatasvir and sofosbuvir for 24 weeks without ribavirin in cirrhotic patients who failed first-generation protease inhibitors," Infection, vol. 45, no. 1, pp. 103-106, 2017.

[22] Hepatitis C treatment Epclusa approved in Canada-key information, CATIE-Canada's Source for HIV and Hepatitis C Information, http://www.catie.ca/en/catienews/2016-07-20/hepatitis-c-treatment-epclusa-approved-canada-key-information.

[23] "Epclusa Recommended by CADTH Canadian Drug Expert Committee If," 2017, http://www.hepctip.ca/drug-pipeline-2/epclusa-recommended-canadian-drug-expert-committee/.

[24] "Harvoni generic entry, patent expiration dates - GILEAD SCIENCES INC: ledipasvir; sofosbuvir," 2017, https://www.drugpatentwatch.com/p/tradename/HARVONI.

[25] "Sofosbuvir; velpatasvir generic drug entry, pharmaceutical patent expiration and FTO," 2017, https://www.drugpatentwatch .com/p/generic-api/SOFOSBUVIR\%3B+VELPATASVIR.

[26] D. Moher, A. Liberati, J. Tetzlaff, D. G. Altman, and The PRISMA Group, "Preferred reporting items for systematic reviews and meta-analyses: the PRISMA statement," PLoS Medicine, vol. 6, no. 7, Article ID e1000097, 2009. 


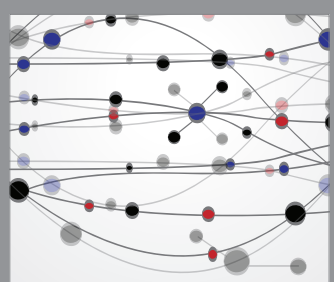

The Scientific World Journal
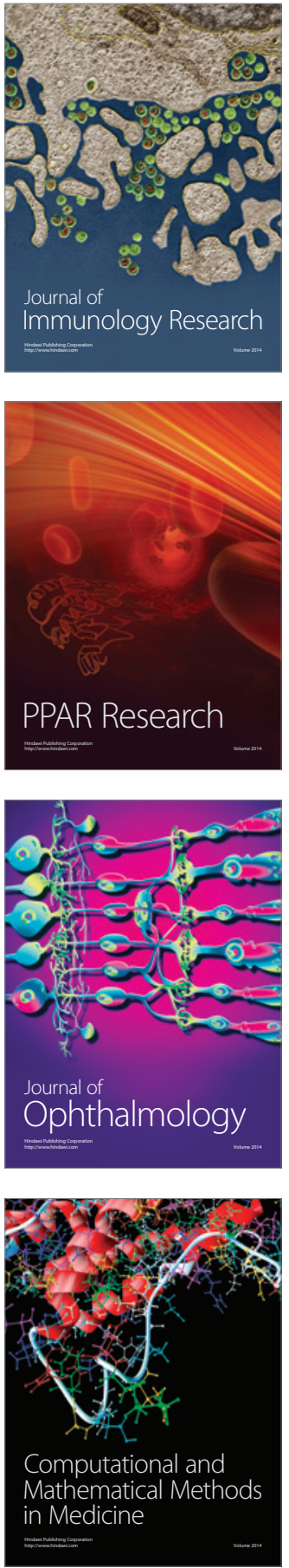

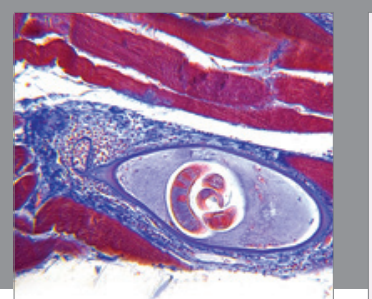

Gastroenterology Research and Practice
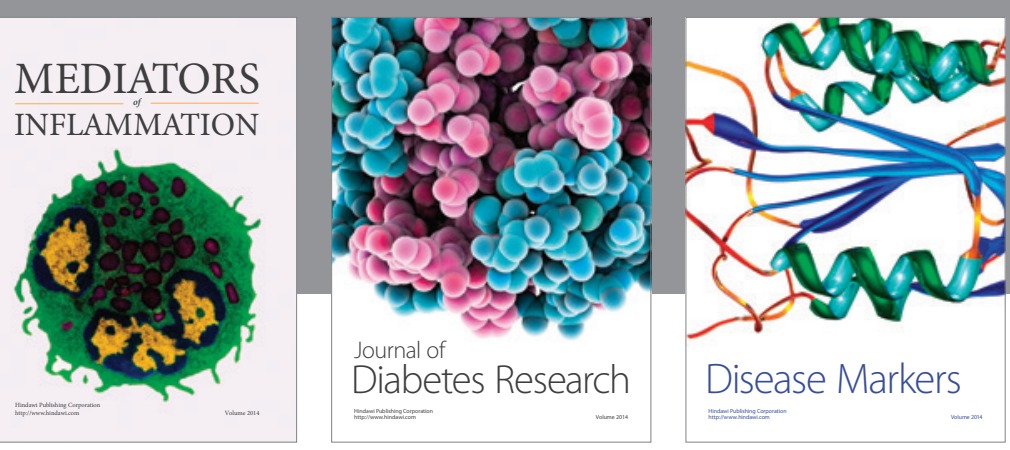

Disease Markers

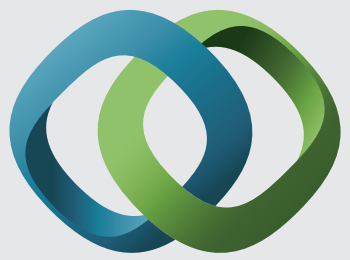

\section{Hindawi}

Submit your manuscripts at

https://www.hindawi.com
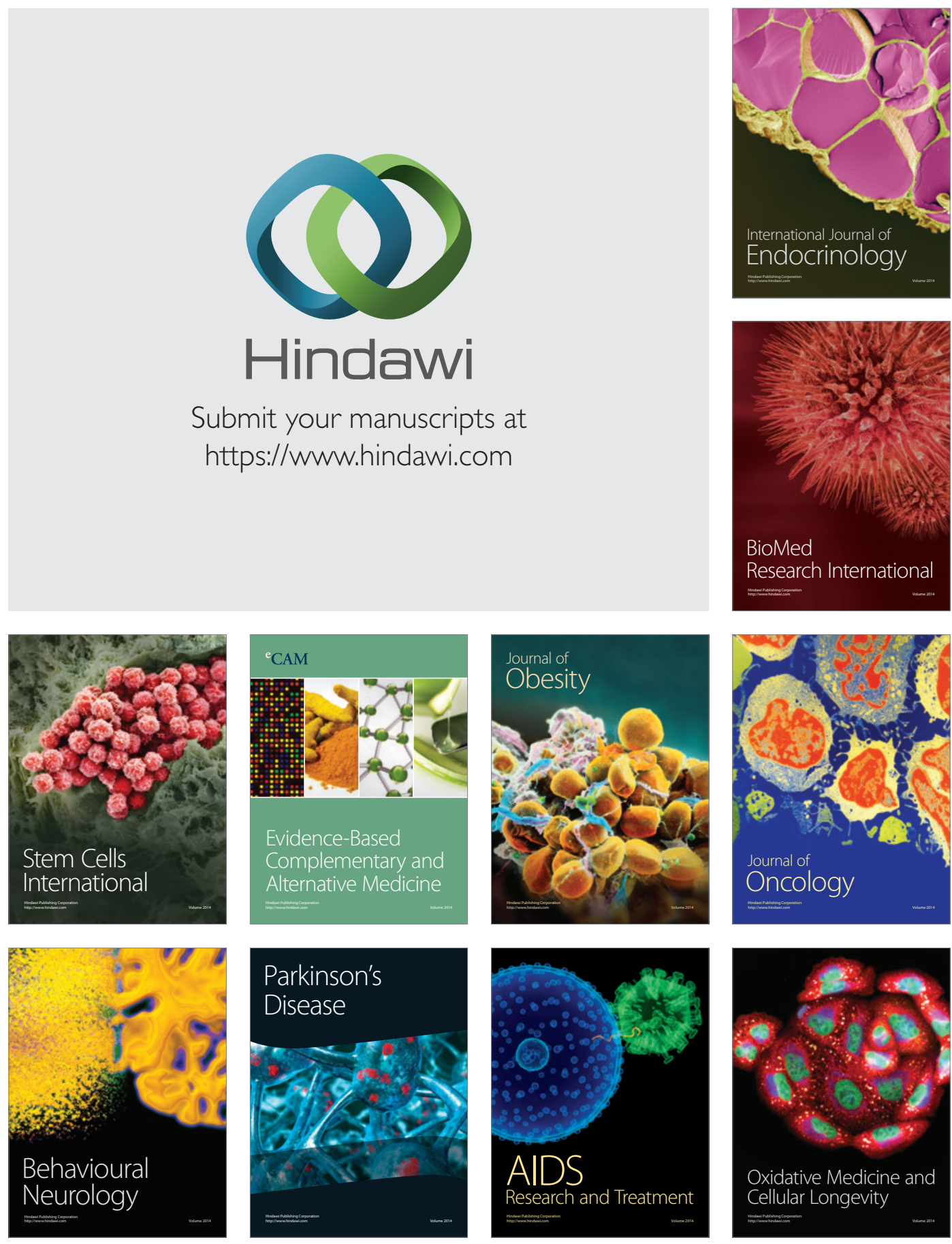